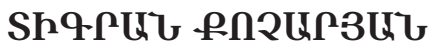

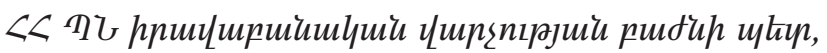

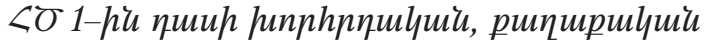

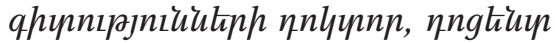

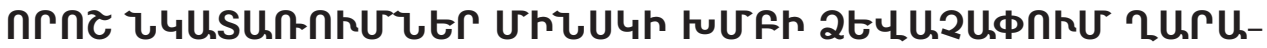

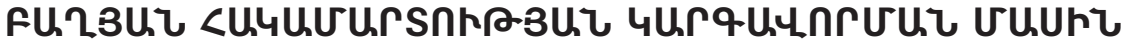

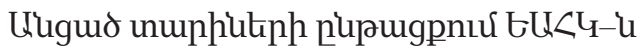
huintru tiluul Uplitiph no Unlứnıunph uhol uhquqqujhi junцuonıpum pnt-

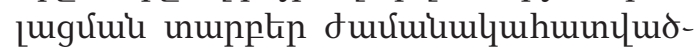
'utinnıu’ quinument punupulquil juju

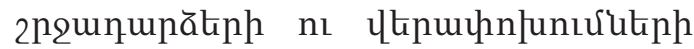

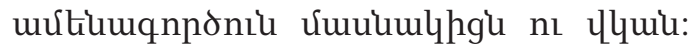

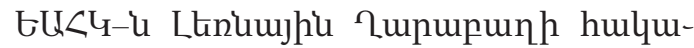

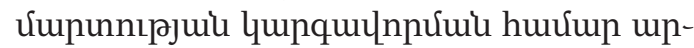

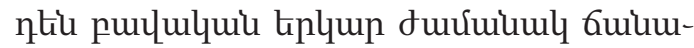

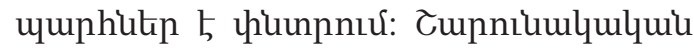
tiu bU<4 Uhiuulh pứph hưumumpumquhitiph unupudu2nquiuujhu ujgknn, npnig dưưuiuml unuing lnnर्uhg hpulqu-

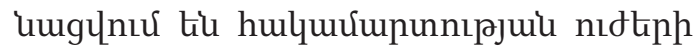

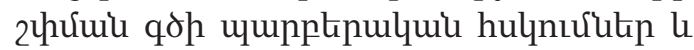

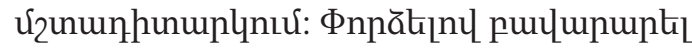
hulquưupunn

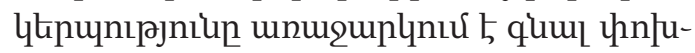
qhqưui qnponn úpquqqujhi unpútiphu

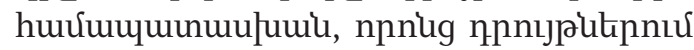
quiu ulyhujun hulquunıpjniukten (Uqqk-

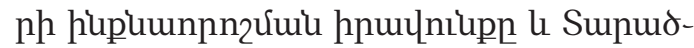
pujhu ứpnngulumunıpjnıun):

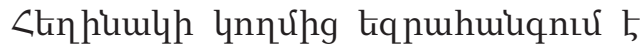

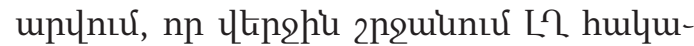

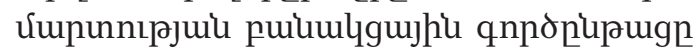

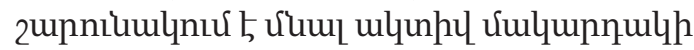
Цnu, hisn, umlumju, sh huightginıu hu-

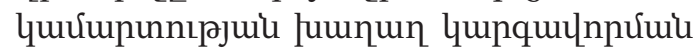
hungntu bulquiu unuqnupugh: <ujlqu-

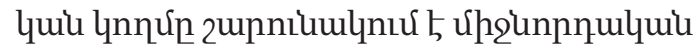

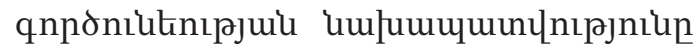

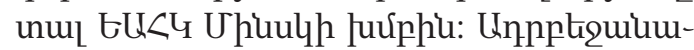

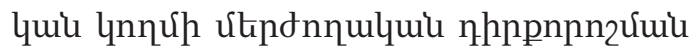
umunfumnl úpginnnulquiu pn!np uwum-

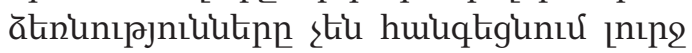

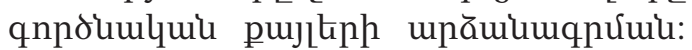
blnnumuluiu undthuưumunqnuर्u «wquunnıpjniun" hn punănuqnuju untinu niuh,

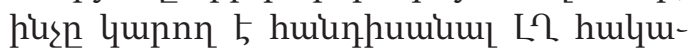

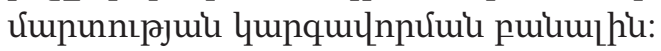

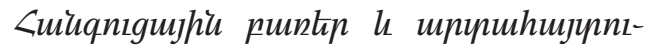

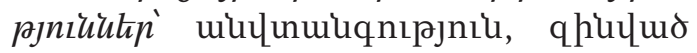
nıdtin, hulquर्umunnıpjnil, цnпर्u, puuwugnıpjnilukin, wquin hupunnn2nıu, unupuдpujhi uúpnngulquinıрjnıl, juununnıpjnilu, uhginnnulumi unuptinıpjnilu:

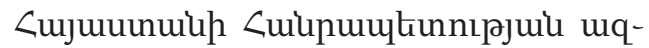

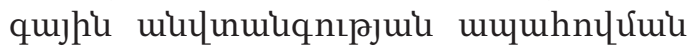

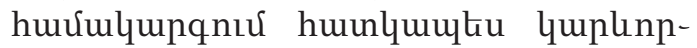

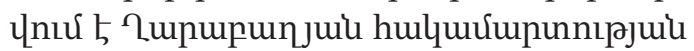

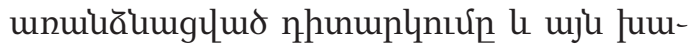
nun Guiumumphy hupptins uumunu-

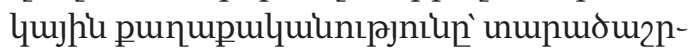

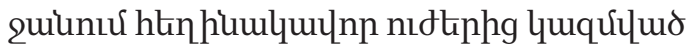
Uhuulh hứph hưर्uरumunumup uumunu-

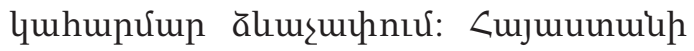

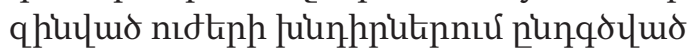

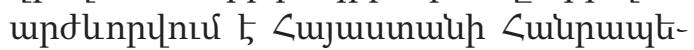
unnıjuiu la Ltenumjhi Lupupunh <uiu-

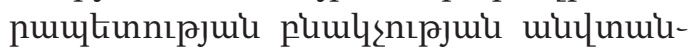

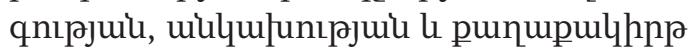

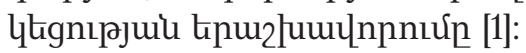

Ltinuwjhu hupupunh hulquưupunnt-

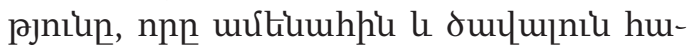

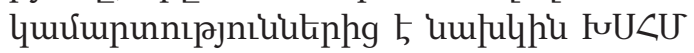




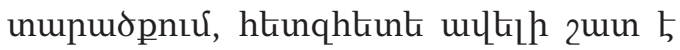
ătinp phinnuর ăqăqumঠ úpquqqujhu $\mathrm{l}$ uhoukunulyuil fuinnh punsp’ qunquguwu ns huonuly htenuilyupitipnl: Ulqq-

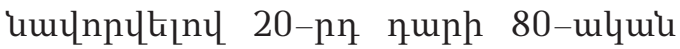
plumuluknh цtinghu (ntinku fuU $<U$ punupulquil unupudpntu)' uju $\mathrm{f} U \angle U$

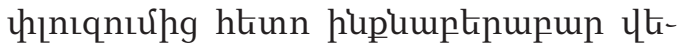
nudylig kplns unpulumuru ukinnıpjniu-

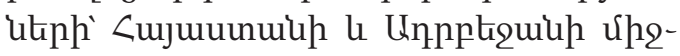
ukinulyuu juinnh:

Ujuuntin untinhi 5 'uthlqujughtil 2unupunjuil hulquर्umpnnıрjuil duqumil úp hnpp umunumlquil mliump:

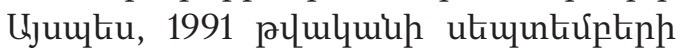

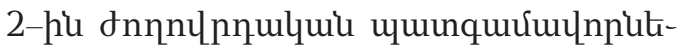

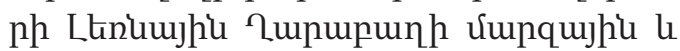
zuhnưjuiuh 2nquiuujhi lunphnıpnuteph hưưunkn uuunurnguin hnswulkg Lknuwjhi hupupunh Cuinumbinnıpjnı-

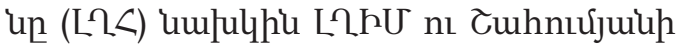

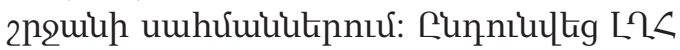

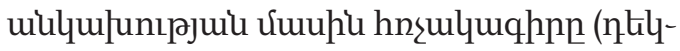
jupughu): Gরumu pujınl hpulquiumgltig

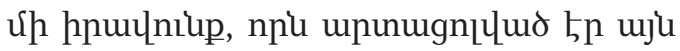

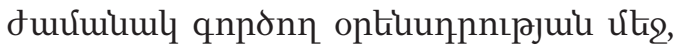

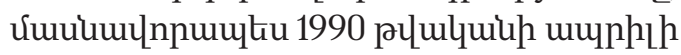

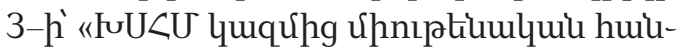

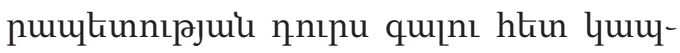

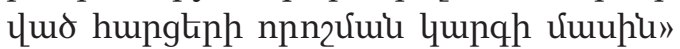

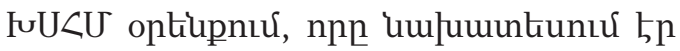

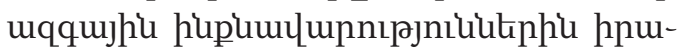

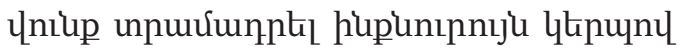
nnn2kins ukhulqui ukinupnululume

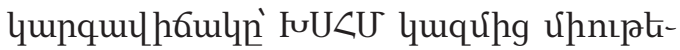

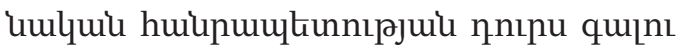

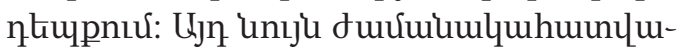
ठnıर्u (1991 plulyuuh unjtưptinhu), h hu-

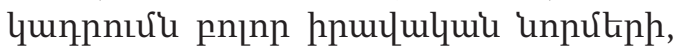
Unpptiquiu qtinuqnuju lunphnınnp nu-

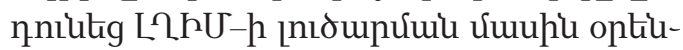

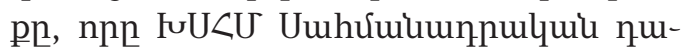
unupuiuh lnnर्uhg npwlyltg npultu $\mathrm{fuU} \cup U$ Uuhưuiumnnıрjuin hulquunn whun:

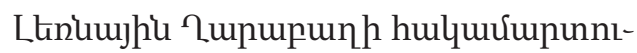

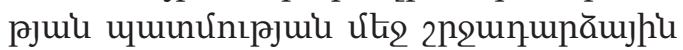
tпuц 1988 pluquiun, tipp Ungurup on-

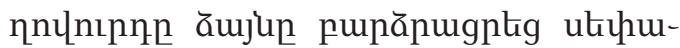
quiu hpuцniuputiph no uquunnıрjuiu

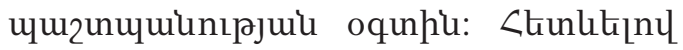
qnnonn pninn hpuцulquiu unpưtiphi $\mathrm{l}$

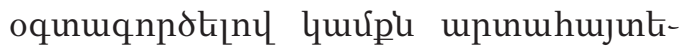

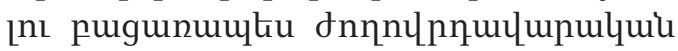
ălikp' Ltnhumph Lupupunh huj uqqupuwl¿nıpjniup huiuntu tiquel <ujuunu-

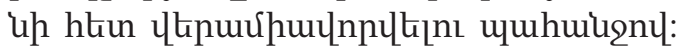

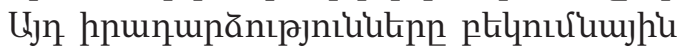
tnuiu ne úpuju ungurughitiph ljuiupntu. npuip, huuunnptiu, umpumuwhưuitighu

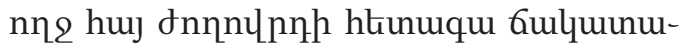
qhnn: 1988 plulquilh hkinnцunh 20-hu

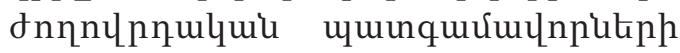

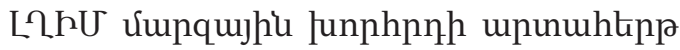
uunuznguiun npn2nư punniukg, npu hp

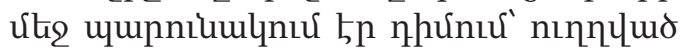
Unpptiquih qtipuqnuju junphnphì 'unu

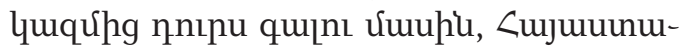
uh qtenuqnuju junphpnhu' unu luwqú utig unitint umuph b foU $\angle U$ qtipuqnuju

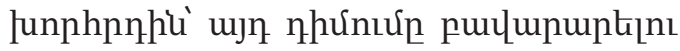

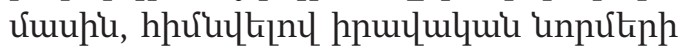

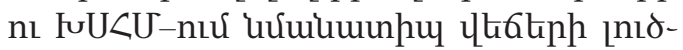

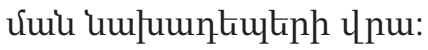

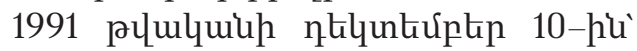

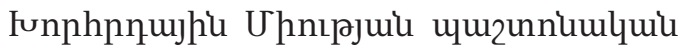

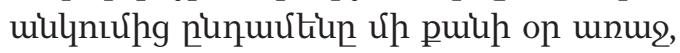

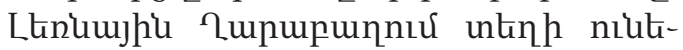
gul huinuplth, nph pupugpnuर uqqu-

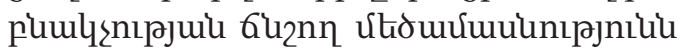
unnumujunltig Unpptiquing inhl wuquifumiumens oqunhis: গpuil htionluwd

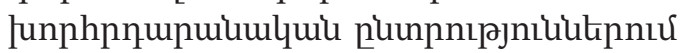

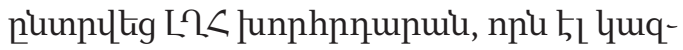

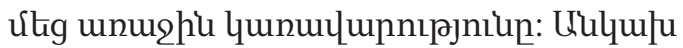

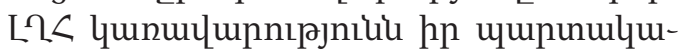
unıpjniuktiph hpulquiumgumulu wigur

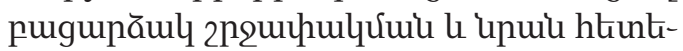

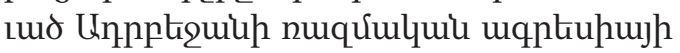

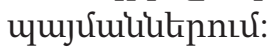


Oqunuqnpotinl $\mathrm{hp}$ unupudpnuर

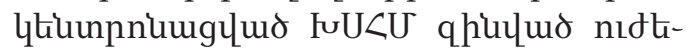

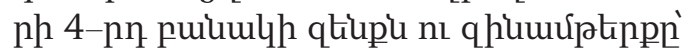
Unpptrquin jujuudulul umuntpuqưu-

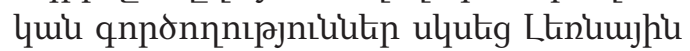

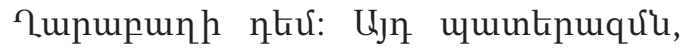

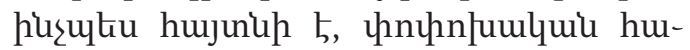

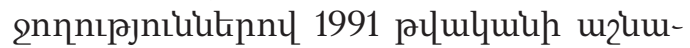
uhg zupnitumlylth uhusl 1994 plulyulup umjhup: Enuiu uwhtp, thp L? unupudph qptipt 60 unnlynup qnulluo bp, hul

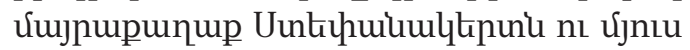
puwlyulujptipn qptipt ulinunup hptunulyndnứukph nı núpulynonứuteph thu Łipuplyцnıर:

1992 pumulum vujhupi L $2<$ hipuu-

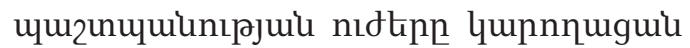
uquinuqptil Znı2h punupp, Uhouigp "pughi" Lushiu punuph 2nquintư hum-

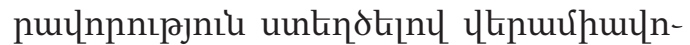

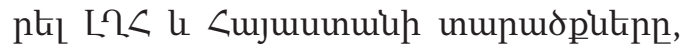

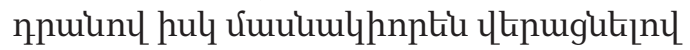

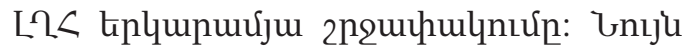

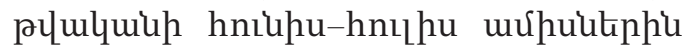
unpptrquiulyuil puiumbir husnnytg

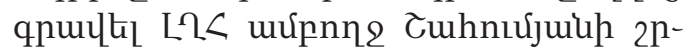
quin, Uupunulthpun 2nguilh vito vuun, Uupunniuns, Uultipuiup le <umpnıph 2nguiutuph uh umup:

Unpptrquh uqptipujhì nhर्umlujt-

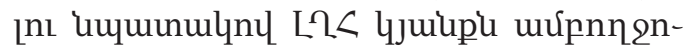

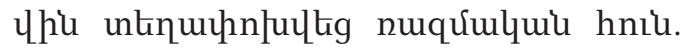
1992 plulquil oqnuinnup 14-his untind-

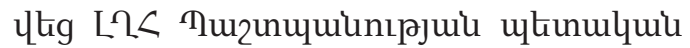

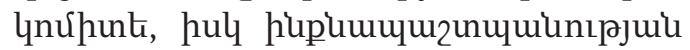

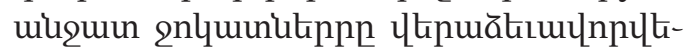

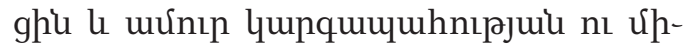

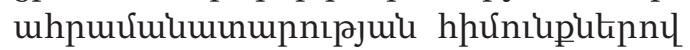

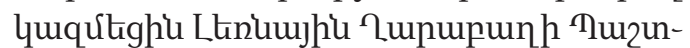

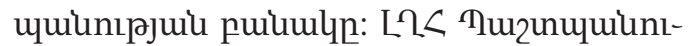
pjuil puiumlihi husnnytig uquinuqpil Unpptrquil lnnर्uhg wlthl lun 2ngu-

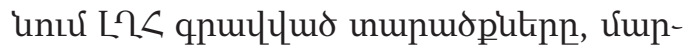
unuluiu qnponnnıpjnitukph pupugpniর

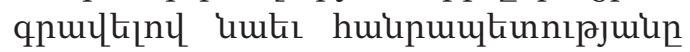

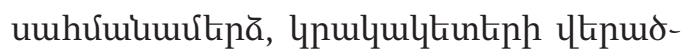

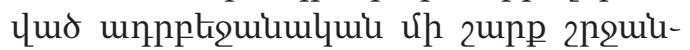

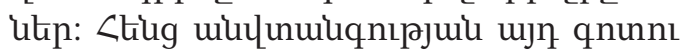

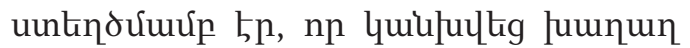

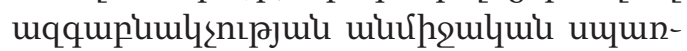
uwihph humpulnpnıpjniun: rul 1994

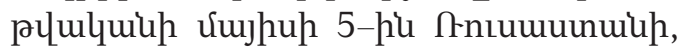

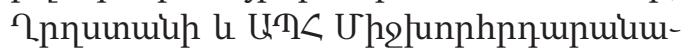

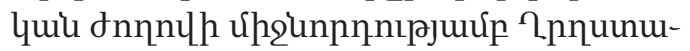
uh vujnupunup Fh2ptilnuर Unnptiquin, Ltinkujhi Rupupunn l. Cujuuunuin unnpuqptighu fh2ptyjuil upăuluuqpnıpjniun , nph hhर्uwiu lpu uujhuh 12-his

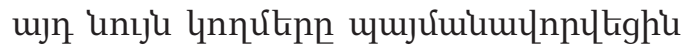

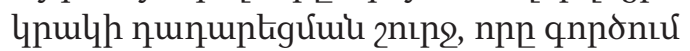
५ uhus ujuon:

Uhquqqujhi huipnıpuulu winniuhg ¿upupunjulu hulquर्umpnnıрjuil lupquцnpưứ thuumugh lhuqnplud bp

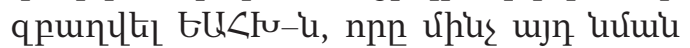

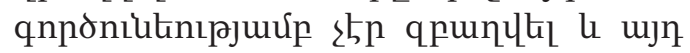

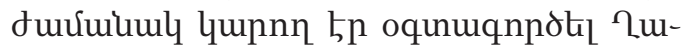

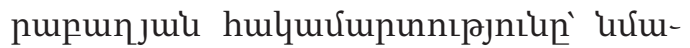

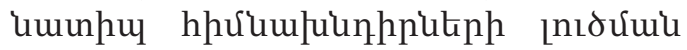
puuquulunnıर hp htinhuulynıpjniun

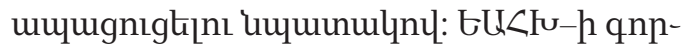
onitiknıpjnilup Rupupunjuil hulu-

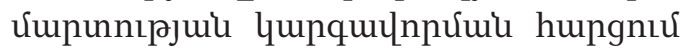

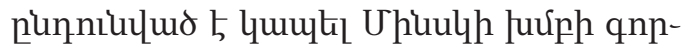
onitunıpjuil hku, npp unuppkp quuhumulquiutiph 5 upduiumg ti: Uhiulh

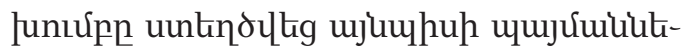

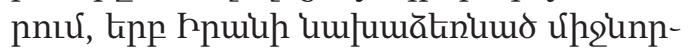

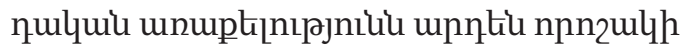

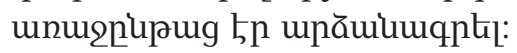

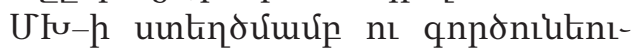
pjưर्य u2lumph unuquinup ntpuquinnıрjnilu nitugnn kplpitinn, pptiug

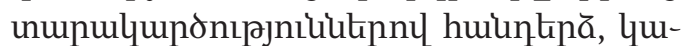

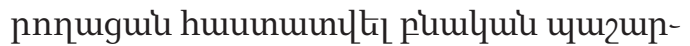
utipny hupniuu Uplứnıunph le Uplutiph

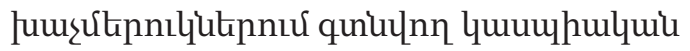
unupudu2pquintu: २uujud 1992 рцuquilh hnikumph 30-hu <ujuuunuiup le

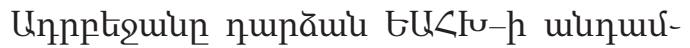


utip l EU<to qnnonn umpuwquhhiu jün-

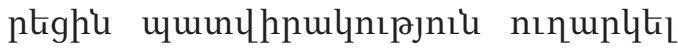

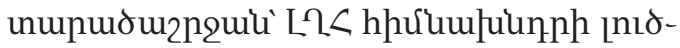

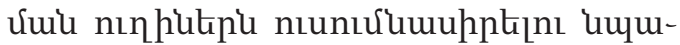
unulynul, umlyuju úpquqqujhu huinnspjuil utnqnuц Uuiun Unpptiquin hting ulqphg puguumpun mpămquiptig,

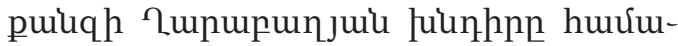

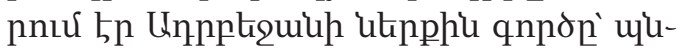

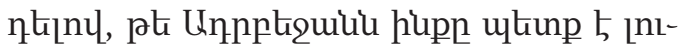
oh ujn hungn:

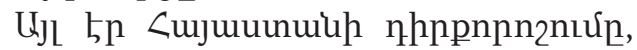

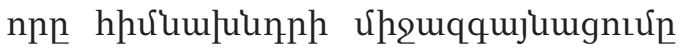

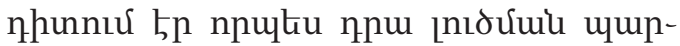

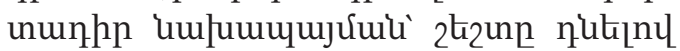

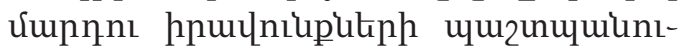
pjuil le wqqkiph uquin hupunnnरuu

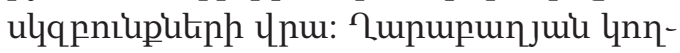

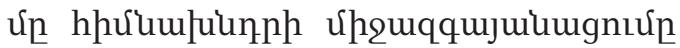

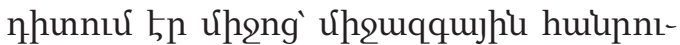

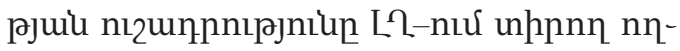

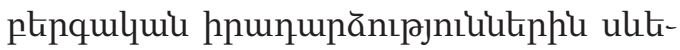

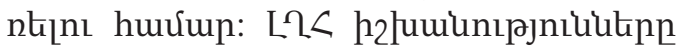
hungh úhquqqujumgưuúp hnpănuর bhu

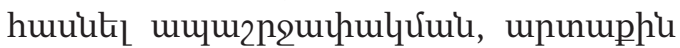

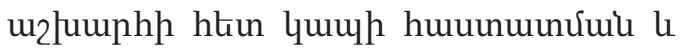

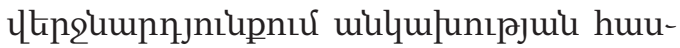

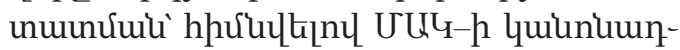

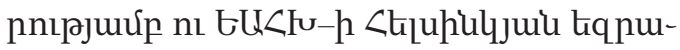

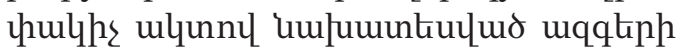
wquun hupunpnzưuiu ulqpniuph цnu:

Lupupunjui hulquर्umunnıرui

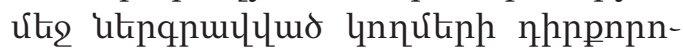

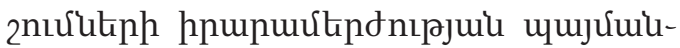

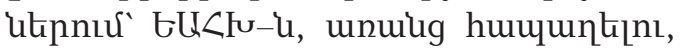
oqunuqnetig unwehu hul humpuln-

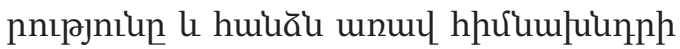

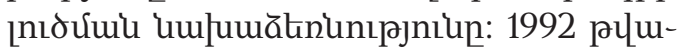

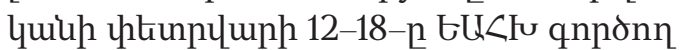
umpumquhh huiuăumpupnıрjứp quq-

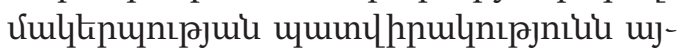

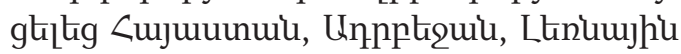
2upupun, nphg htiunn qtilnusg tukplu-

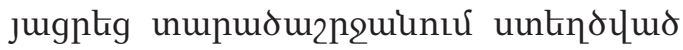
hnuulh6ulh umuhu [2]:

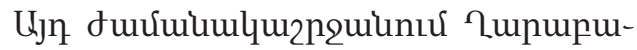

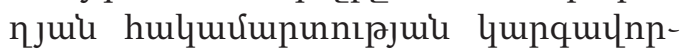

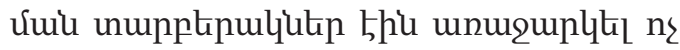

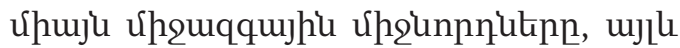
qhonulumu 2nquiumluteph utiplujugnt-

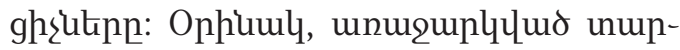

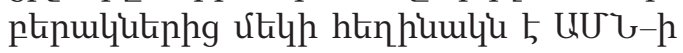

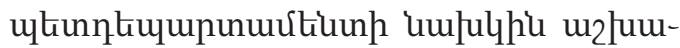

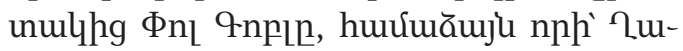

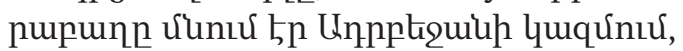
hul <ujuuunuin nteup hupupun tip

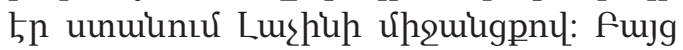

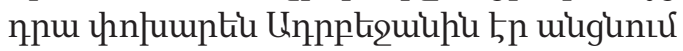

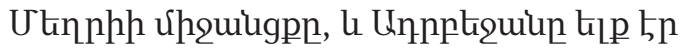

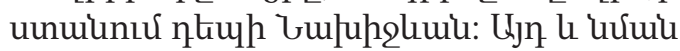

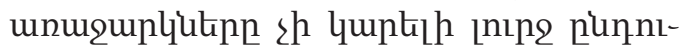
ukl, puiu np npuip úpuizuiuml wink-

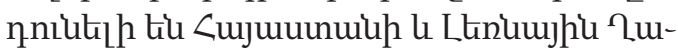
nupunh hưump:

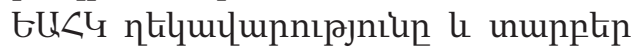

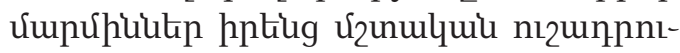

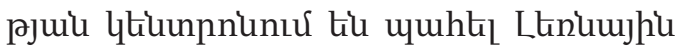

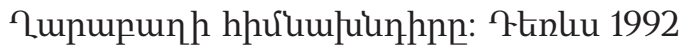

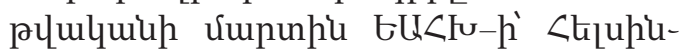

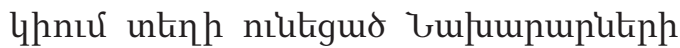
lunphpnh uhuunnuर L? hulquưupunnt-

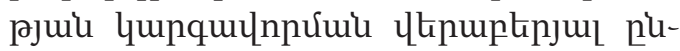

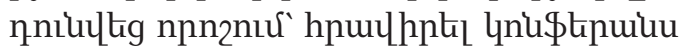
Uhuulnıu: Gni\$thumul umpumquh

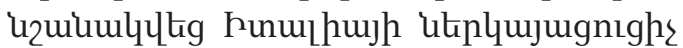

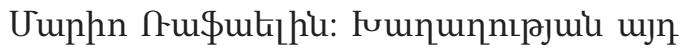

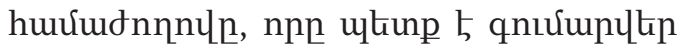

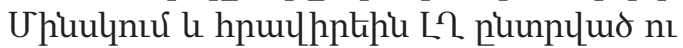

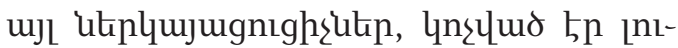

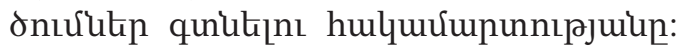

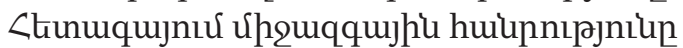

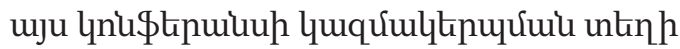
uuniunl qnpopupugu no punqplyum ukunnıpjniuutinhu lynstig Uhiuuh junıúp:

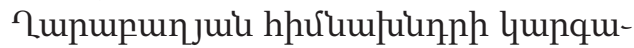
unpưuu unwehi ulqqpniupp Cujuuunu-

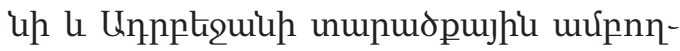

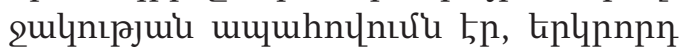

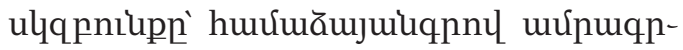

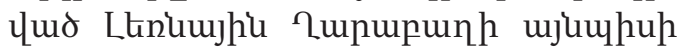




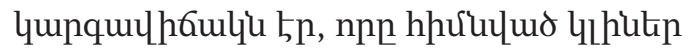

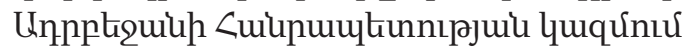

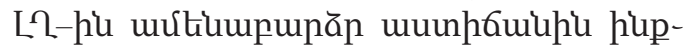

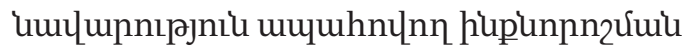

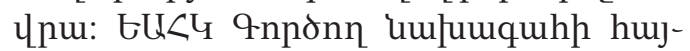
unupnıpjuiun útluntin thumpnujuil huil-

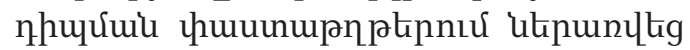
<ujuuunulh uquinlhpulnцрjuiu hujununupnıpjniup, npunte ưumhnqnıрjniu $5 n$

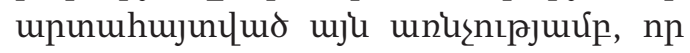
ঢU<4 Uhuulh luúph umlumquhp hujununupnıpjniun sh upunugn!nư Uhiulh bứph ưưnumn nqhu, hųn tiupunpnıu 5 puiumlgnıpjnilutep punupulquil huưwămjunıpjui huuktent umuunulnul:

4unquuh6ulh hpúlumluunhpn puumplưulu unuplqu 5 kntil nınhn puuulgnıpjniuutent pupugpnuर, npnup

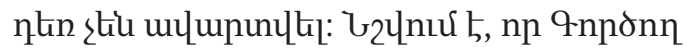
uwhumquhh hujununupnıpjnikn quilun-

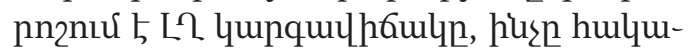

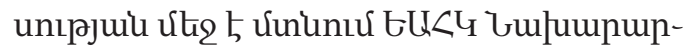
uknh lunnhpnh 1992 plulquiuh npnzưui htiu, npn ujn hungn ylenuwumbl $5 p$

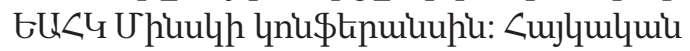
unnứ hưưnqứnıup 5 upunuhujunnıर्u, np

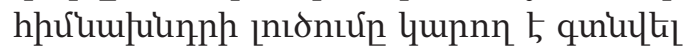

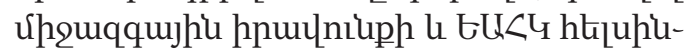

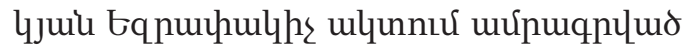

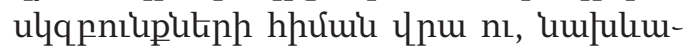
nuq̀ uqqtiph hupunnnzưu ulqpniuph:

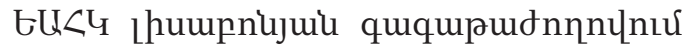

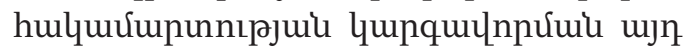
puiumăliu hn hhúpniu nithp uwe hhर्u-

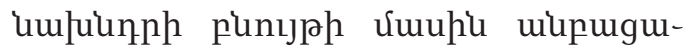

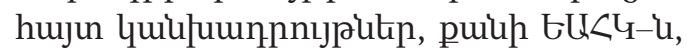

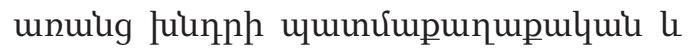

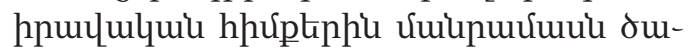
unpuiument, Ltinuwjhi hupupunn nhunnuर $5 p$ Unpptrquiph umunluminn unu-

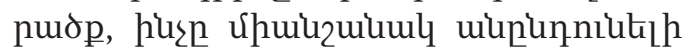
ununkgnư $5 n$ [3]:

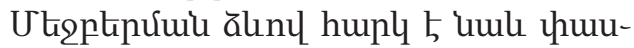

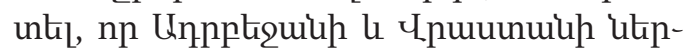

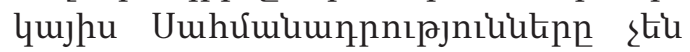

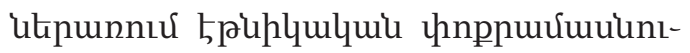

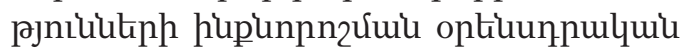
unpuktp: Spliug uwhưuiumpnulyuiu optiupuknny ujn ukinnıрjnilutinn úhuiưưu tiu b umpulhi bunphnnujhu hupumump huipuwukinnıpjniukteph' Uplumqhujh,

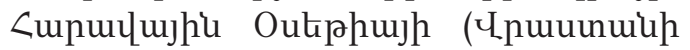
Uuhưuuunnnıрjuiu 1-hu qliuh 2-pn

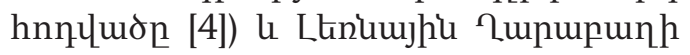

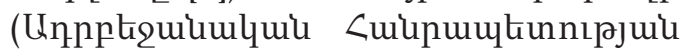

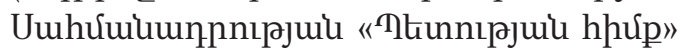

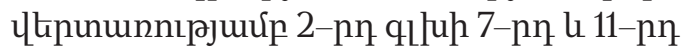

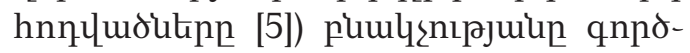
umlquinnu pnul \&til unulhu pumlıntpjuu 2nquiunıu hujun utpplujughtinı $h p$ ukplujhu pumlnnpjuil umjnknnıर hip-

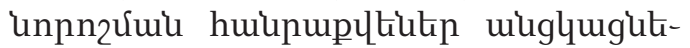

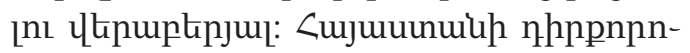

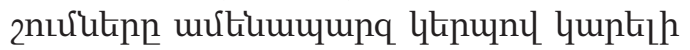

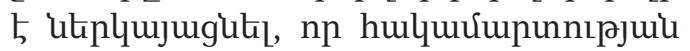

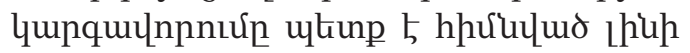
htinlujul ulqqpniuputiph unw. Lteniuujhi ¿upupunh hulquर्umunnıрjuiu lupqu-

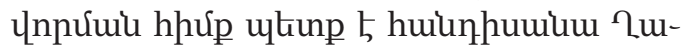
nupunh dnnnцlnnh hupunpnzưuu hpu-

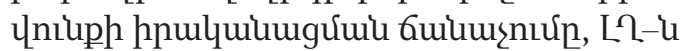
<ujuunuilh htion ulting 5 nilitium huj-

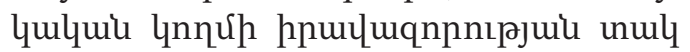

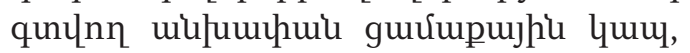

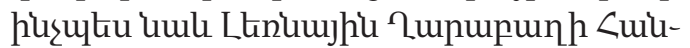

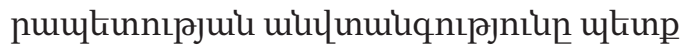

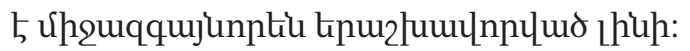
Uju ulqqpniupukph punniunıর'u nı uщj-

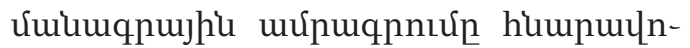

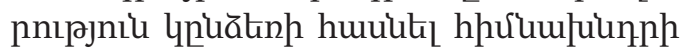
huruurhp yunquulnnर्umiun:

Unnupugh 5 vouuumqtiuktinh lnnuhg 2upupunjuil hulquर्umunnıрjuil

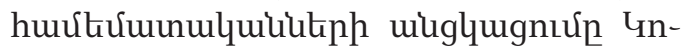

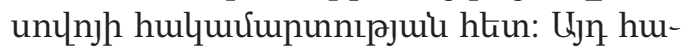

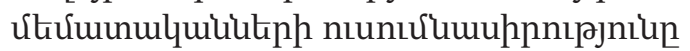

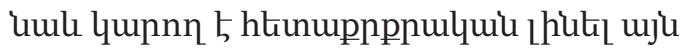

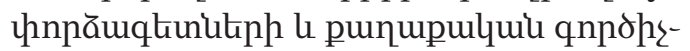
ukph hưưun, npnup unpănư tiu ưưunıpjnitukip quitul <upuulujhu Gnunцn- 


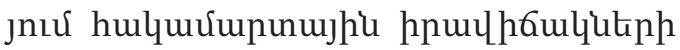

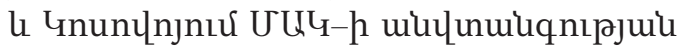
junphpnh npn2nıúutiph gnujup utippn

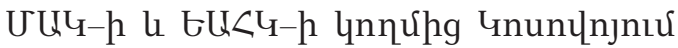

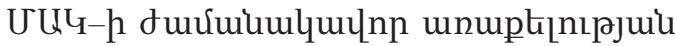
nı $\quad \mathrm{U}<<\mathrm{4}-\mathrm{h}$ unuptinıpjuil uhengny qnnдunluwo hupunnn2nıưujhu qnp-

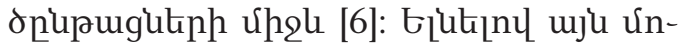
unkgnuxhg, np hưưzlumphujhu undt-

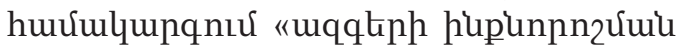
hnulniupn" hp punăpuqnıju unțu

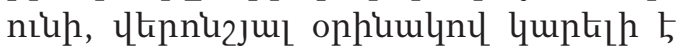
lu úhquqqujhi huipnıpjniup ukinp 5

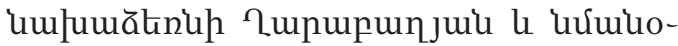
nhiuwl ujnıu hulquưupunnıjnılukteph unpupugh qunquunnưul qunnıgu-

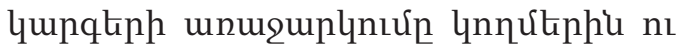
hnuqnnonıú:

กpuku Lkniuughu 2upupunh Cuiunuwkinnıрјui (Unguruh) pumlınıрјui

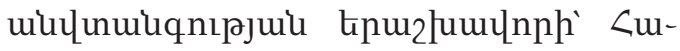
juunuiuh <uipumiknnıpjuil mqqujhi

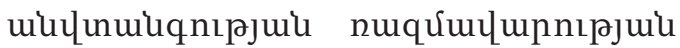
utig Ltniumph Rumpupunh hulqu-

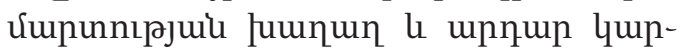
quцnnर्umu fuinhpi nilh unuiggpujhi

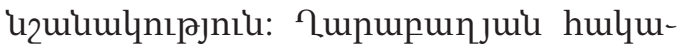

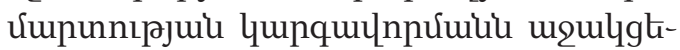

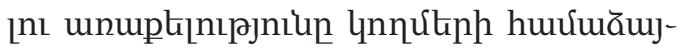
unıрנuи́p lumuhltil 5 EU<4 Uhuulh

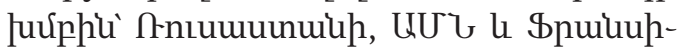
ujh huर्umum|umquhnıрјuर्up: Cuјшu-

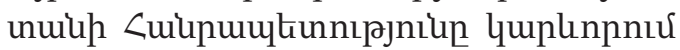

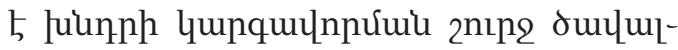

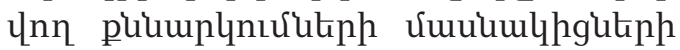

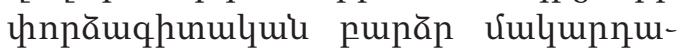

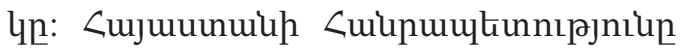

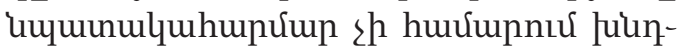

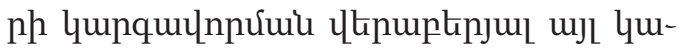
nnıgukinh lnnuरhg hujunupupnıpjniukt-

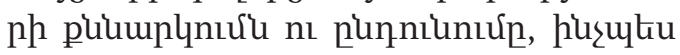
umb uhguqqujhi unupptip luquiulykn-

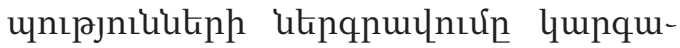

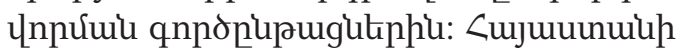

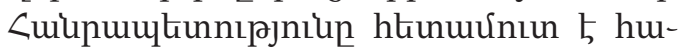

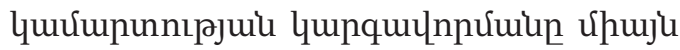

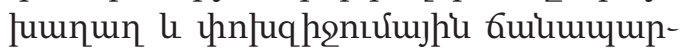
hnl: Ltiniuujhi Rupupunh Cuipumb-

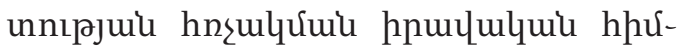

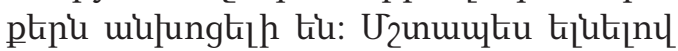
uju ulqpniuphg, np guilqugur lthp'uu-

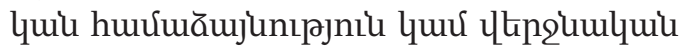

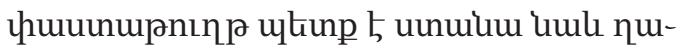

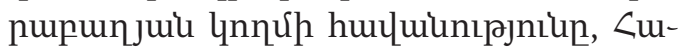

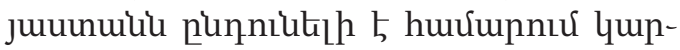
quunnưuil úpuju uju unupptinulutinn,

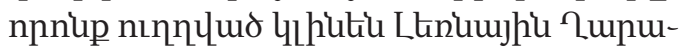
punh <uinumulinnıpjuiu huuunugh qn-

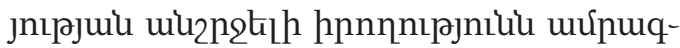
ntįnıi [7]:

2umjud $\mathrm{GU<4}$ Uhuulh bưph 2n-

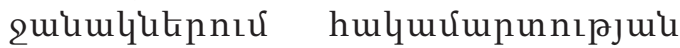
4unquunnर्umu únguqqujunptiu pu-

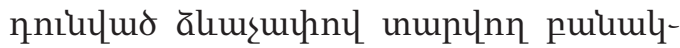
gnıpjniuutiphu l npuip Uhuulh qnpopupugh 2nquiumlikinnu pupuiument

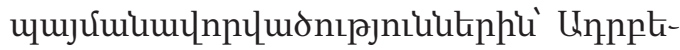

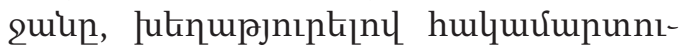

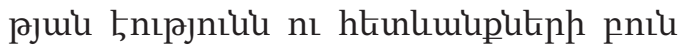

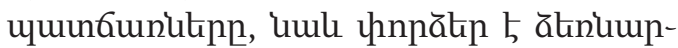

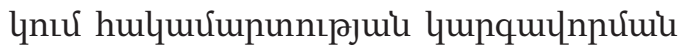

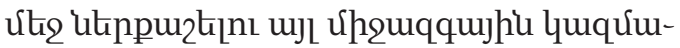

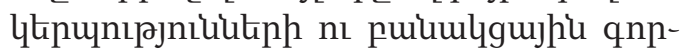

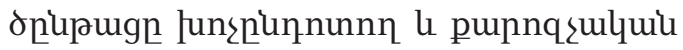
uumunulytin htinuwinnn qnıquhtin

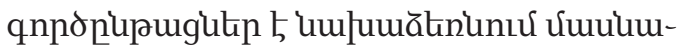

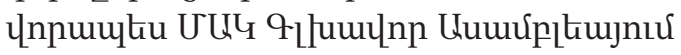
nı Elnnuwjh Junphnnnuर:

Unpptiquiuh цunwor nuqưumbius uq-

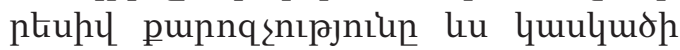
unul 5 nunıर फnfuqhonıर्ujhu qunqu-

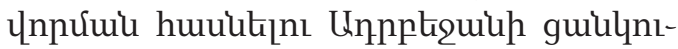
pjnıun, hujunupupnıpjnitukinn, uunuuă-

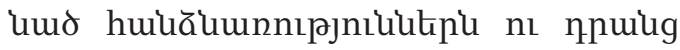

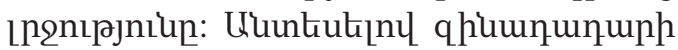

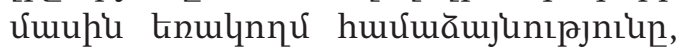
Unpptiquin úphr opu sh nunupnu qu-

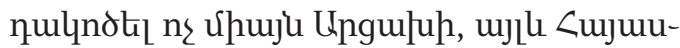

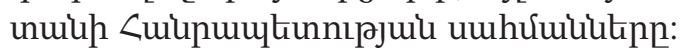

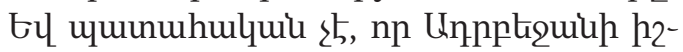




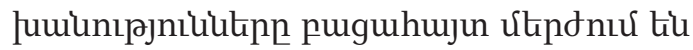

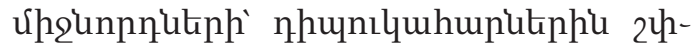

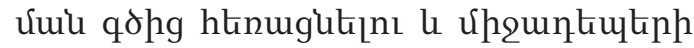

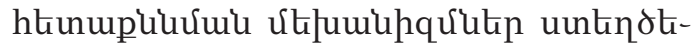
jnı unuquplitinn, inıjuhul hpudup-

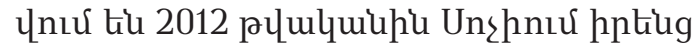
hul lnnuरg uunnumquluo hujununu-

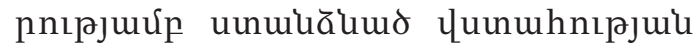

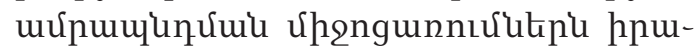
qnpðtinıg [8]: Unpptrquin uulpujhi tquर्unıuntinhg unugumo qnıर्uplut-

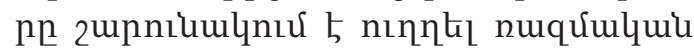

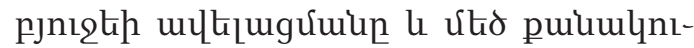

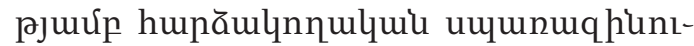

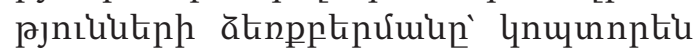

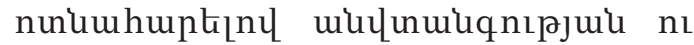
nuquupunupulqui n!npunh uh zwnp hưưămjumqptip le umpunulnpnopjniuitip:

Ujuinkn hupl 5 punqdtal, np hhuumbuinnp qunquulnpưui nuqưupunupulyui qhonultipןnıdulquil htiuph

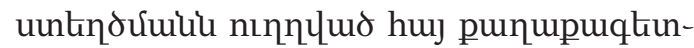
ukph quiptenn hhưlumlquiunư huiuqnuर

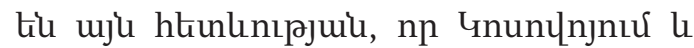

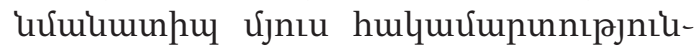

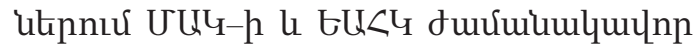

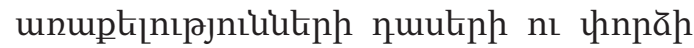

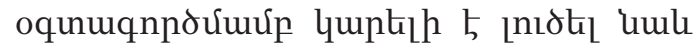
Uplumqhujh, Cupulujhi Outphujh li

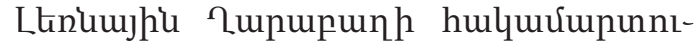

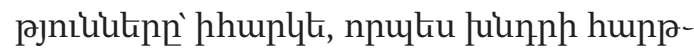

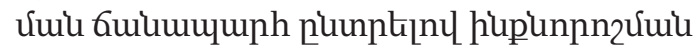

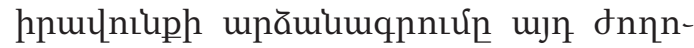

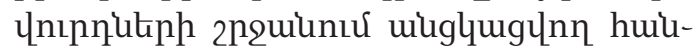
pupltiukph úpgngnu [9]:
Luin 5nıрjuiu, Unpptiquin ипшщш-

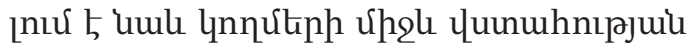

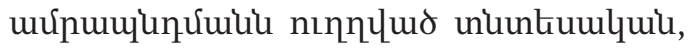
punupulquil, nuqualqui no úmpu-

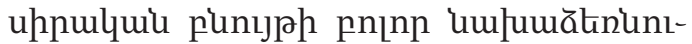
pjnilutinn: Uuulumunnumbu, Unpptiquin

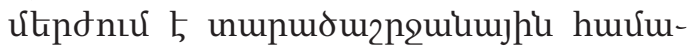

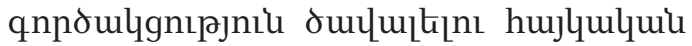

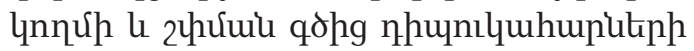
htinpwzưuu Uhuulh bứph hưưumbum-

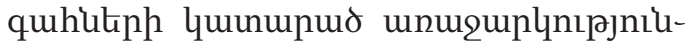
utinn: El hhwplt, nnuktu tiqnuhwuqnuर nunqdunuर 5, np 2upupunjuiu hulquúununnıрjuil úuuhi junutilhu hupluuцnn 5 hulquर्umunnıpjnikn nhunupltki ununudurnquiumpi le úpruqqujph qunqu-

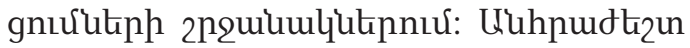

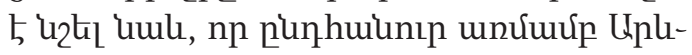

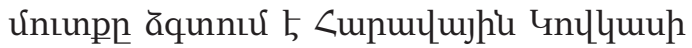

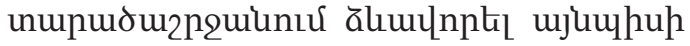
huuunuunnıjnilutip le utpnitil ujuuhup

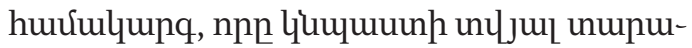
дurnquiunú hiunkqpughni qnpopipaugukph huunuunnilu qunqugúuiun: Lknuujhi Lupupunh hulquर्umunnıpjniuu ujuon huinhpumiuntú 5 numudurnguiup

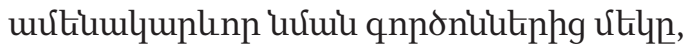

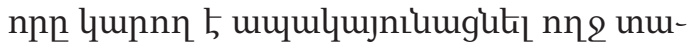

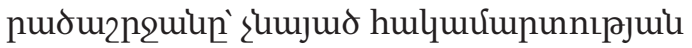
hungniu status-quo-h guilqugurd

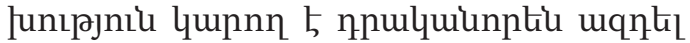
huj-pnıppulqui hupuptennıpjniuutiph lnu, npi bl hp htipphi juul humunuцnnnıpjnilutip quuntindp <unuцujhi 4nцlumunuर huuntiqnughnu ஓnuqpinp hưump:

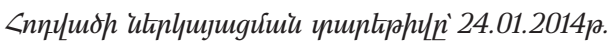

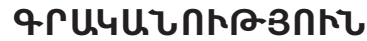

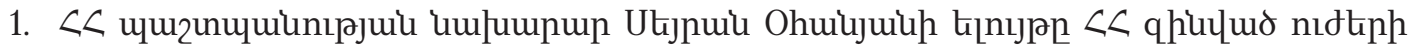

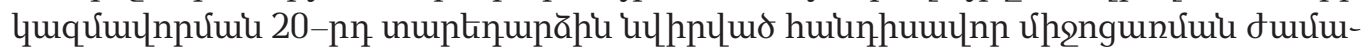
uwl, 27.01.2012p., http://www.mil.am/1327666346/page/35

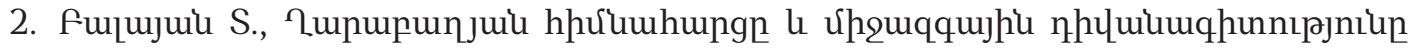
1991-1994 pцulquiutiphu, tn., 2004, 5254

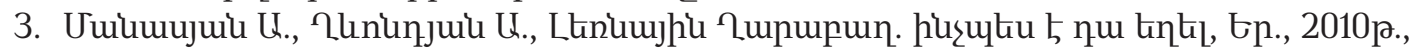
52 155-156 
4. http://members.tripod.com/ggdavid/georgia/const8.htm

5. http://www.constitutional-court-az.org/const-chapter2.htm

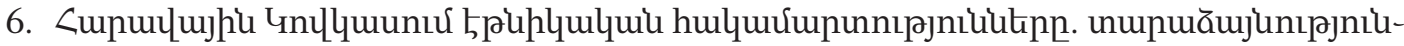

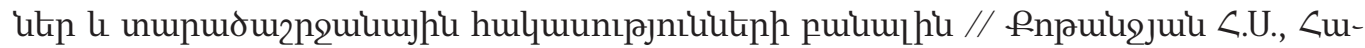

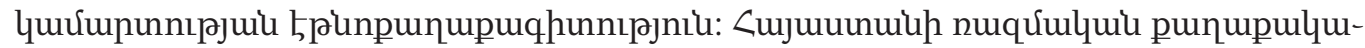

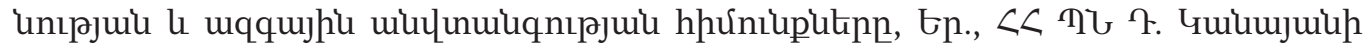
wuluiu Un $\angle \mathrm{H}, 2010$, 52 663:

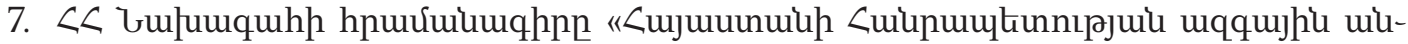

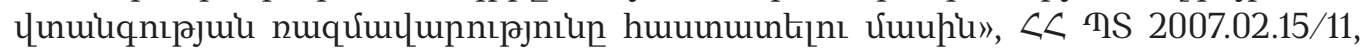
07.02.2007, 3-pn qlnıłup

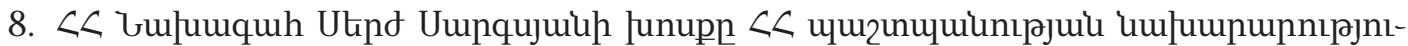

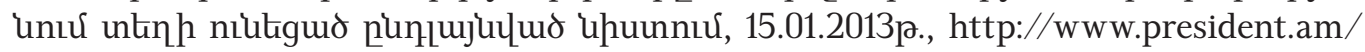
hy/statements-and-messages/item/2013/01/15/President-Serzh-Sargsyan-speech-session-Ministry-of-Defense/

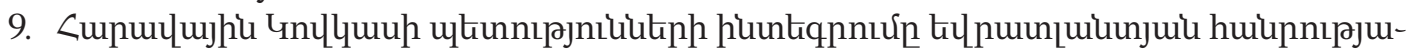

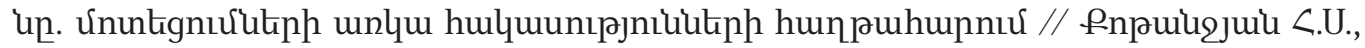

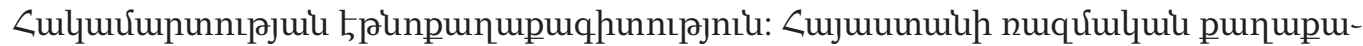

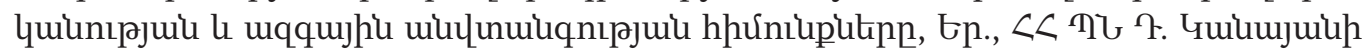
uiluluiu Un<L, 2010, 52 681:

\section{SOME CONSIDERATIONS ABOUT KARABAKH CONFLICT SETTLEMENT IN THE FORMAT OF THE MINSK GROUP}

\section{TIGRAN KOCHARYAN}

Head of the Branch of the Law Department of the Ministry of Defence of RA, 1-st Class Adviser, Doctor of Political Sciences, Associate Professor

The separated monitoring of the Nagorno-Karabakh conflict and the intended policy for peaceful regulation are very important in the system of providing national security of the Republic of Armenia in more acceptable format of the Minsk Group. During the regional visits of the Minsk group co-chairs are being realized regular controlling and monitoring the contact line of the conflicting forces. Tryaing to satisfy the conflicting parties, the Minsk Group offers a compromise according to the international norms, in which are some obvious discrepancies (The right of nations to self-determination and The territorial integrity). Recently, the regulation process of the Nagorno-Karabakh conflict remains in the active level, which, however, does not lead to substantial progress in the peace process. The Armenian part keeps giving priority of the intermediary activity to the OSCE Minsk group. Because of the negative position of the Azerbaijan part all intermediary initiatives are not leading to serious practical results. The freedom has the main place in the European value system, which can be the key for the regulation of the Nagorno-Karabakh conflict. 


\section{НЕКОТОРЫЕ СООБРАЖЕНИЯ О РАБОТЕ МИНСКОЙ ГРУППЫ ПО УРЕГУЛИРОВАНИЮ КАРАБАХСКОГО КОНФЛИКТА}

\section{КОЧАРЯН ТИГРАН}

Начальник отдела юридического управления Министерства обороны РА, Советник

1-ого класса, доктор политических наук, доцент

В системе национальной безопасности Республики Армения особенно подчеркивается отдельное рассмотрение нагорно-карабахского конфликта и целевая политика по мирному регулированию конфликта в более приемлимом формате Минской группы. В ходе региональных посещений соопредседателей группы осуществляются регулярный контроль и мониторинг линий соприкосновения противоборствующих сил. Пытаясь удовлетворить конфликтующие стороны Минская группа предлагает пойти на компромисс в соответствии с существующими международными нормами, в которых есть очевидные противоречия (право на самоопределение наций и территориальная целостность). В последнее время переговорный процесс нагорно-карабахского конфликта продолжает оставаться на активном уровне, который, однако, не приводит к существенному прогрессу в мирном регулировании конфликта. Армянская сторона продолжает уделять приоритетное внимание посреднической деятельности Минской группы ОБСЕ. Из-за негативного отношения азербайджанской стороны все посреднические инициативы не приводят к фиксированию практических результатов. В европейской ценностной системе “свобода” имеет особенное место, которая послужит основным ключом к регулированию нагорно-карабахского конфликта. 